Res Balticae 1998, pp. 117-129.

\title{
THE LANGUAGE OF THE OLD PRUSSIAN CATECHISMS*
}

\author{
FREDERIK KORTLANDT \\ Leiden
}

Elsewhere I have presented a comparative analysis of the three Old Prussian catechisms (1998). I first put together the three texts which translate the same German original. The collation will be reproduced here for the sake of reference.

\begin{tabular}{|c|c|c|}
\hline I (1545) & II (1545) & E (1561) \\
\hline Staey dessempts & Staey dessimpts & Stai Dessimton \\
\hline Pallapsaey. & Pallapsaey. & Pallaipsai. \\
\hline Pirmas. & Pirmois. & $\begin{array}{l}\text { Stas Pirmois } \\
\text { Pallaips. }\end{array}$ \\
\hline $\begin{array}{l}\text { Thou ni tur kittans } \\
\text { deiwans tur- } \\
\text { rettwey. }\end{array}$ & $\begin{array}{l}\text { Tou ni tur kittans } \\
\text { deiwans tur- } \\
\text { ryetwey. }\end{array}$ & $\begin{array}{l}\text { Tou niturri kittans } \\
\text { Deiwans pagâr } \\
\text { mien turrîtwei. }\end{array}$ \\
\hline Anters. & Anters. & $\begin{array}{l}\text { Stas Antars } \\
\text { Pallaips. }\end{array}$ \\
\hline $\begin{array}{l}\text { Thou ny tur schan } \\
\text { emnen twaise deiwas } \\
\text { ny anterpinsquan } \\
\text { menentwey }\end{array}$ & $\begin{array}{l}\text { Tou } n i \text { tur sten } \\
\text { emnen twayse dey- } \\
\text { was nienbæenden } \\
\text { westwey. }\end{array}$ & $\begin{array}{l}\text { Tou turri stan } \\
\text { Emnan twaisei Dei- } \\
\text { was ni enbândan } \\
\text { westwei. }\end{array}$ \\
\hline Tirts. & Tirtis. & $\begin{array}{l}\text { Stas Tîrts } \\
\text { Pallaips. }\end{array}$ \\
\hline $\begin{array}{l}\text { Thou tur schan } \\
\text { lankenan deinan } \\
\text { swintintwey. }\end{array}$ & $\begin{array}{l}\text { Tou tur stan } \\
\text { lankinan deynan } \\
\text { swyntintwey. }\end{array}$ & $\begin{array}{l}\text { Tou turri stan } \\
\text { Lânkinan Deinan } \\
\text { Swintint. }\end{array}$ \\
\hline
\end{tabular}

This contribution was presented at the 8th International Congress of Baltic Linguistics, Vilnius, October 1997. 
Ketwerts.

Thou tur twaian thawan bha muttin somonentwey.

Penckts.

Thou ny tur gallintwey.

Wuschts.

Thou ny tur pattiniskun lembtwey

Sepmas.

Thou ny tur rancktwey.

Asmus.

Thou ny tur falsch widekausnan waitiatun preiken twaien tawischen

Newints.

Thou ny tur pallapsittwey twaisei tawischis buttan.
Ketwirtz.

Tou tur twayien

thawan bhæ mutien smunintwey.

Pyienkts.

Tou ni tur gallintwey.

Vsts.

Tou ni tur salobisquan

limtwey.

Septmas.

Tou ni tur ranktwey.

Asmus.

Tou ni tur reddi weydikausnan waytiaton preyken twayien tauwyschen.

Newyntz.

Tou ni tur pallapsitwey twaysis tauwyschis butten.
Stas Kettwirts

Pallaips.

Tou turri twaian

Tâwan bhe Mûtien smûnint.

Stas Piêncts

Pallaips.

Tou niturri gallintwei.

Stas Vschts

Pallaips.

Tou niturri

Sallûbanlimtwei.

Stas Septmas

Pallaips.

Tou niturri

Rânctwei.

Stas Asmus

Pallaips.

Tou niturri reddewijdikausnan dâtwei prijki twaian tawischan.

Stas Newînts

Pallaips.

Tou ni turri

Pallaipsîtwei, twaisei tawischas buttan. 
Dessimts.

Thou ny tur pallapsittwey

twaisei tawischis gennan, waikan, mergwan, pecku, ader katanassen asch.

Stas Dröffs.

AS drowe an Deiuan, Thawan wismosing, kas ast taykowuns dangon bah semmin, Bah an Jesum Christû swaian ainan Sunun nusun rekian, Kas patickots ast assastan swintan naseilen, Gemmons assastan jungkfrawen $\mathrm{Ma}-$ rian. Stenuns po Pontio Pylato, scrisits, aulawns bah encops. Sammay lesuns preipekollin, Tirtin deinam att skiwuns assa gallans. Vnsey gobuns andangon, Sindats preitickray Deiuas wismosingis Thawas,
Dessympts.

Tou ni tur pallapsitwey twaysis tauwyschies, gennan, waykan, mergwan, pecku, adder katanæssen hest.

Stan Druwin.

ES drowy en Deywan, Thawan wissemokin, kas est tykynnons dængon bhe semmien.

Bhæ en Jesum

Christum swaien aynen Sounon nouson reykyen, Kas pagauts æst hæse swyntan naseylien. Gemmons æsestan jungprawan Marian. Styienuns po Pontio Pylato, skresitzt, aulauns bhe enquoptzt. Semmay lysons prey pykullien, An tirtien deynan etskyuns hæse gallans. Vnsei gubons nadengon. Syndens preytickaray deywas wyssen mukis thawas,
Stas Dessîmts

Pallaips.

Tou niturri

pallapsitwei

twaise Tawischas Gannan, Waikan, Mêrgan, Peckan, adder ka tennêison ast.

Stas Swints Cristiâniskas Druwis.

As druwê en Deiwan, Tâwan Wissemusîngin kas ast teikûuns, Dangon bhe semmien. Bhe en Jesûm Christum, swaian ainan Soûnon noûson Rikijan, kas pogauts ast, esse Swintan Noseilien, Gemmons esse stan Jumprawan Marîan, Stînons po Pontio Pilato, Skrîsits, Aulauns, bhe enkopts, Semmailisons prei Pickullien, Entîrtan deinan etskîuns esse gallan, Vnsaigûbons no Dangon, Sîdons prei tickrômien Deiwas steise wissemusîngin Tawas 
Stwendau pergubuns wirst preyleiginwey stans geiwans bha aulauwussens.

As drowe an swintan naseilen, Ainan swintan krixtianiskun kirkin, Ainan perroniscon swintan, Et werpsannan grecon, At skisenna menschon, $\mathrm{Ba}$ prabitscun geiwin. Amen.

Sta Thawe nuson.

THawe nuson kas

thu asse andangon. Swintints wirst twais emmens.

Pergeis

twais laeims.

Twais quaits

audasseisin

na sem-

mey key audangon.

Nusan deini-

nan geittin dais

numons schin-

deinan. Bha

atwerpeis noumans

nuson auschautins,

kay mas

atwer pimay
Stwendau wirst pergubons leygenton stæns geywans bhe aulaunsins.

Es drowy en swyntan naseylien, Aynan swyntan krichstianisquan kirken, Perronisquan swyntan, Etwerpsennian griquan, Etskysnan menses, Bhe prabusquan geywien, Amen.

Stan Thawe nouson.

THawe nouson kas thou æsse æn-dengon, Swyntits wirse tways emmens, Pareysey noumans tway ia ryeky, Tways quaits audaseysin

nasemmiey kay endengan, Nouson deyninan geytiey days noumans schian deynan. Bhæ etwerpeis noumans nouson anschautins, kay mes etwerpymay isquen dau tâns pergûbons wijrst, prei lîgint stans gijwans bhe aulausins.

As Druwê ênstan Swintan Noseilien ainan Swintan Cristiâniskan kijrkin Stan perôniskan Swintan etwerpsennian steise grijkan etskîsnan mensas, bhe ainan prâbutskan gijwan, AMEN.

Stas Tâwa Noûson.

Tâwa Noûson kas tu essei Endangon.

Swintints wîrst twais Emnes.

Perêit twais Rijks. Twais Quâits Audâsin, kâgi Endangon tijt dêigi nosemien. Nouson deinennin geitien dais noûmans schan deinan. Bhe etwerpeis noûmas nousons âuschautins, kai mes etwêrpimai 


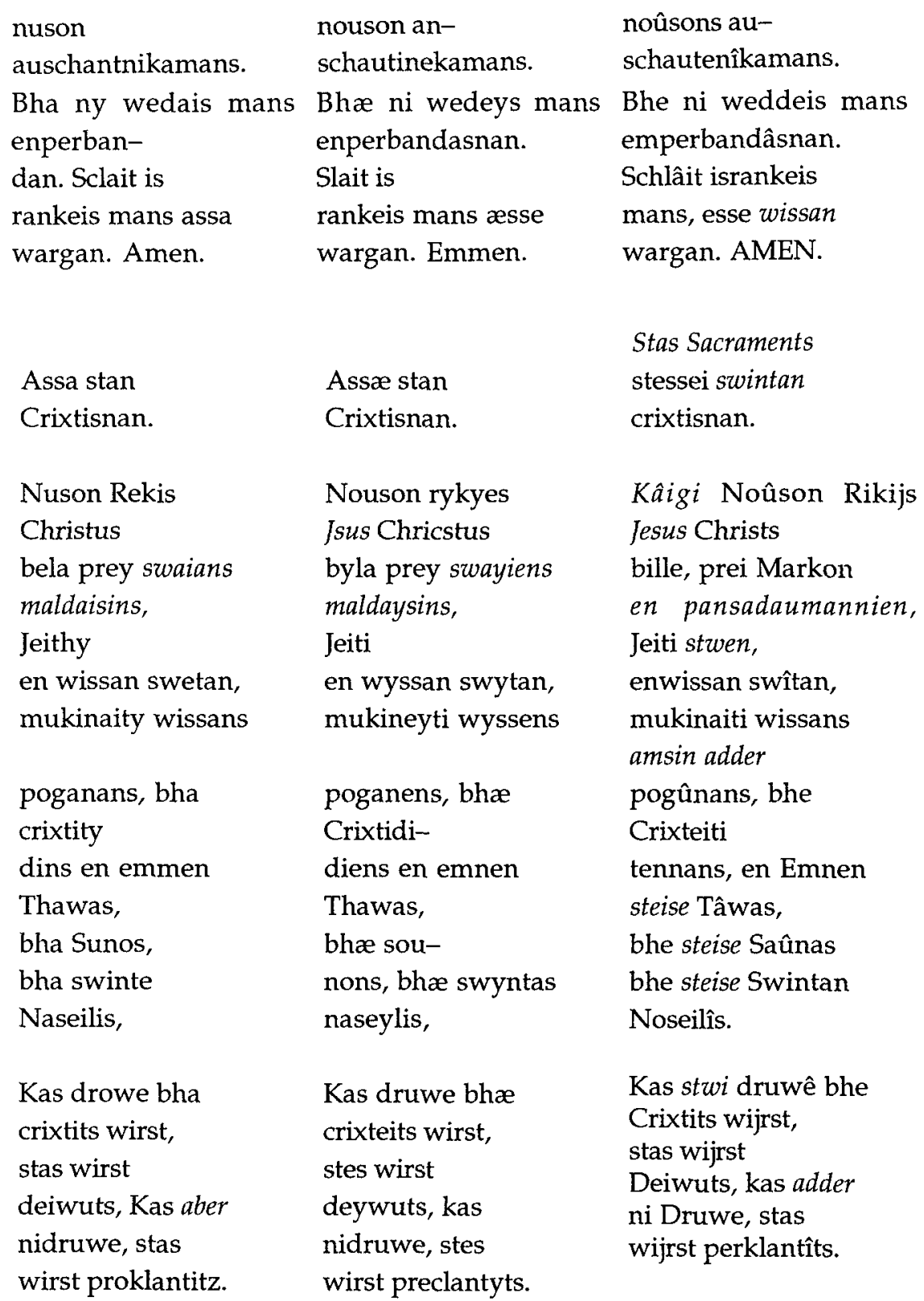




\begin{tabular}{|c|c|c|}
\hline $\begin{array}{l}\text { Assa Sacramentan } \\
\text { bietis eden. }\end{array}$ & $\begin{array}{l}\text { Assa Sacramenten } \\
\text { bytis ydi. }\end{array}$ & $\begin{array}{l}\text { Stas Sacraments } \\
\text { Steisei Altari. }\end{array}$ \\
\hline $\begin{array}{l}\text { Nusen rickis } \\
\text { Jesus Christus, } \\
\text { anstan nactin } \\
\text { kadan } \\
\text { proweladin, } \\
\text { ymmits stan } \\
\text { geittin, dinkowats } \\
\text { bha limatz bha } \\
\text { daits swaimans } \\
\text { maldaisemans bha } \\
\text { belats, ymaity } \\
\text { stwen, edeitte, } \\
\text { sta ast mais ker- } \\
\text { mens, kas } \\
\text { perwans dats } \\
\text { wirst, staweidan } \\
\text { segeitty } \\
\text { prey mayan } \\
\text { menissnan. }\end{array}$ & $\begin{array}{l}\text { Nouson reykeis } \\
\text { Jsus Chricstus } \\
\text { anstan naktin } \\
\text { kaden } \\
\text { proweladin, } \\
\text { ymmeits sten } \\
\text { geytien, dinkautzt } \\
\text { bhe lymuczt, bhe } \\
\text { dayts swaymans } \\
\text { maldaysimans, bhe } \\
\text { bylaczt, ymayti } \\
\text { stwen, bhe ydieyti, } \\
\text { stæ æst mays ker- } \\
\text { mens, kas } \\
\text { perwans daeczt } \\
\text { wirst, Steweyden } \\
\text { segeyti } \\
\text { premayien me- } \\
\text { nisnan. }\end{array}$ & $\begin{array}{l}\text { Noûson Rikijs } \\
\text { Jesus Christus, } \\
\text { ênstan Nack- } \\
\text { tien, kaden tans } \\
\text { prawilts postâi, } \\
\text { imma tans stan- } \\
\text { geitin dînkauts } \\
\text { bhe lîmauts, bhe } \\
\text { dai swaimans, } \\
\text { maldaisimans, bhe } \\
\text { billâts, immaiti } \\
\text { stwen, îdeiti, } \\
\text { sta ast mais } \\
\text { kêrmens, kas } \\
\text { pêrwans dâts } \\
\text { wîrst, Sta- } \\
\text { wîdan seggîtei } \\
\text { prei maian } \\
\text { pominisnan. }\end{array}$ \\
\hline
\end{tabular}




\begin{tabular}{|c|c|c|}
\hline Stasma polleygo & Stæsmu poleygo & Stesmu empolijgu \\
\hline jmmitz deyg & ymmeyts deygi & immats dijgi \\
\hline stan kelkan pho & sten kelkan, & stan Kelkin \\
\hline stan betten eden, & postan bitans ydi, & pobîtas îdin, \\
\hline dinkowatz bha daitz & dinkauczt bhe daits & Dinkauts, bhe dai \\
\hline swaimans & swaymans & stan steimans \\
\hline maldaisemans & maldaysimans & \\
\hline $\begin{array}{l}\text { bha belats, jm- } \\
\text { maitty stwen, bha }\end{array}$ & $\begin{array}{l}\text { bhæ bilats, ymmay } \\
\text { ti stwen bhe }\end{array}$ & $\begin{array}{l}\text { bhe billâts, Jmaiti } \\
\text { stwen, bhe }\end{array}$ \\
\hline pugeitty wissay & puieyti wyssay & poieiti wissai \\
\hline is stasma, schis & istesmu, $\mathrm{Sis}$ & isstesmu, Schis \\
\hline kelchs ast sta & kelkis æst stæ & kelks ast stas \\
\hline nawans testamentan, & neuwenen Test & nauns Testaments \\
\hline an maian & menten en mayiey & en maian \\
\hline kraugen, kha & kræuwiey, ka & krawian, kas \\
\hline perwans palletan & per wans praliten & perwans pralieiton \\
\hline werst, pray att & wirst, prey et- & wîrst, prei etwer- \\
\hline werpsannan gre- & werpsennian & psennien stêison \\
\hline kun, steweydan & griquan, Stewidan & grijkan, Stawîdan \\
\hline segeitty, kodesnim- & segeyti kudesnammi & seggîtei \\
\hline maian & yian & \\
\hline menisnan. & minicnan & II \\
\hline
\end{tabular}

Then I eliminated the orthographical differences between the three versions while maintaining the distinction between linguistic variants. Finally I assigned separate phonemic interpretations to the three versions on the basis of my review of the historical evidence (1988). I concluded that the three texts basically represent three successive stages in the development of the moribund Prussian language. Here I shall give a systematic account of the main differences between the three versions.

The phonological differences between the three catechisms largely result from the diphthongization of monophthongs and the monophthongization of diphthongs: 
(1) $* \overline{\mathrm{e}}>$ I $[\overline{\mathrm{e}}]>$ II $[$ ie] $>$ E [ī]

I turrettwey, II turryetwey, E turrîtwei.

I stenuns, II styienuns, E stînons.

I lesuns, II lysons, E lisons.

I grecon, II griquan, E grijkan.

I swetan, II swytan, E swîtan.

I bietis eden, II bytis ydi, (E steisei altari.)

I edeitte, II ydieyti, E îdeiti.

I betten eden, II bitans ydi, E bîtas îdin.

I grekun, II griquan, E grijkan.

(2) *ēè > I [ēi] > II, E [iei].

I palletan, II praliten, E pralieiton.

(3) *ēn > I [ēn] > II, E [ien].

I penckts, II pyienkts, E piêncts.

(4) ${ }^{*} \overline{1}>$ I, II [ei] $>$ E [ī]. This close diphthong remains distinct from open *ei $>$ I, II, E [æi].

I widekausnan, II weydikausnan, E wijdikausnan.

I preiken, II preyken, E prijki.

I scrisits, II skresitzt, E skrîsits.

I leiginwey, II leygenton, E lîgint.

I geiwans, II geywans, E gijwans.

I geiwin, II geywien, E gijwan.

I auschantnikamans, II anschautinekamans, E auschautenîkamans.

I crixtits, II crixteits, E crixtits.

I staweidan segeitty, II steweyden segeyti, E stawîdan seggîtei.

I polleygo, II poleygo, E polijgu.

I deyg, II deygi, E dijgi.

I steweydan segeitty, II stewidan segeyti, E stawîdan seggîtei.

(5) $* \bar{a}>*^{\circ}>$ I [uo] $>$ II, E [ū ].

I muttin, II mutien, E mûtien.

I somonentwey, II smunintwey, E smûnint.

I taykowuns, (II tykynnons,) E teikûuns. 
I patickots, (II pagauts, E pogauts.)

I gobuns, II gubons, E gûbons.

I pergubuns, II pergubons, E pergûbons.

I deiwuts, II deywuts, $E$ deiwuts.

(6) ${ }^{*}$ ōi > I [uoi] $>$ II [ūi] $>$ E [oui].

I pugeitty, II puieyti, E poieiti.

I pogeitty, II puietti, cf. E poûis.

(7) ${ }^{*} \overline{\mathrm{u}}>$ I [ü] $>$ II, E [ou].

I sunun nusun, II sounon nouson, E soûnon noûson.

I Thawe nuson, II Thawe nouson, E Tâwa noûson (2x).

I nusan, II, E nouson.

I numons, II noumans, E noûmans.

I, II noumans, E noûmas.

I nuson, II nouson, E nousons.

I nuson, II nouson, E noûsons.

I nuson, II nouson, E noûson.

I sunos, II sounons, E saûnas.

I nusen, II nouson, E noûson.

(8) ${ }^{*} \mathrm{~W}>$ I $[\mathrm{w}]>$ II, E zero before rounded vowels.

I wuschts, II vsts, E vschts.

I taykowuns, (II tykynnons,) E teikûuns.

I aulawns, II, E aulauns.

I att skiwuns, II etskyuns, E etskîuns.

I aulauwussens, II aulaunsins, E aulausins.

but: I deiwuts, II deywuts, E deiwuts.

I dinkowats, II dinkautzt, E dînkauts.

I dinkowatz, II dinkauczt, E dinkauts.

The declensional system was simplified by the elimination of irregular stem forms and endings:

(1) Replacement of i-, $\mathrm{u}$ - and consonant stems by a-stems.

I, II emnen, E emnan.

I tawischis, II tauwyschis, E tawischas.

I tawischis, II tauwyschies, E tawischas.

I, II pecku, E peckan. 
I tirtin, II tirtien, E tîrtan.

I geiwin, II geywien, E gijwan.

(I laeims,) II ryeky, E rijks.

I schin, II schian, E schan.

I dins, II diens, E tennans.

I sunos, II sounons, E saûnas.

(2) Regularization of a-stem endings.

I, II mergwan, E mêrgan.

I krixtianiskun, II krichstianisquan, E crixtiâniskan.

I perroniscon, II perronisquan, E perôniskan.

I grecon, II griquan, E grijkan.

I menschon, II menses, E mensas.

I prabitscun, II prabusquan, E prâbutskan.

I grekun, II griquan, E grijkan.

(3) Elimination of the neuter gender.

I sta Thawe nuşon, II stan Thawe nouson, E stas Tâwa noûson. I sta nawans testamentan, II stæ neuwenen testamenten, E stas nauns testaments.

I kha, II ka, E kas.

The conjugational system was simplified by a reduction of verbal categories:

(1) Replacement of the imperative by the indicative.

I, II tur, E turri (10x).

(2) Replacement of the infinitives in - twey and -ton by -t.

I swintintwey, II swyntintwey, E swintint.

I somonentwey, II smunintwey, E smûnint.

I leiginwey, II leygenton, E lîgint.

(3) Elimination of the nasal infix.

I sindats, II syndens, E sîdons.

(4) Replacement of the optative by the indicative.

I pergeis, II pareysey, E perêit.

I audasseisin, II audaseysin, $\mathrm{E}$ audâsin. 
(5) Replacement of the preterit by the present tense.

I bela, II byla, E bille.

I ymmits, II ymmeits, E imma tans.

I jmmitz, II ymmeyts, E immats.

(6) Replacement of the active by the passive preterit.

I, II proweladin, E tans prawilts postâi.

(7) Replacement of the optative by the imperative? (cf. Kortlandt 1982: 7)

I segeitty, II segeyti, E seggîtei (2x).

There are various discrepancies in the numerals, pronouns and adverbs:

I dessempts, II dessimpts, E dessimton 'ten'.

I pirmas, II, E pirmois.

I tirts, II tirtis, E tîrts.

I schan, II sten, E stan.

I schan, II, E stan.

I dins, II diens, E tennans.

I ymmits, II ymmeits, E imma tans.

I, II stwendau, $E$ isquen dau.

I preiken, II preyken, E prijki.

I vnsey, II vnsei, E vnsai after semmai (Van Wijk 1918: 59).

The prefix and preposition na was replaced by no after po (Van Wijk 1918: 51):

I naseilen, II naseylien, E noseilien (2x).

$\mathrm{I}$ andangon, II nadengon, $\mathrm{E}$ no dangon.

I na semmey, II nasemmiey, E nosemien.

I naseilis, II naseylis, E noseilîs.

The main syntactic innovations are the following:

(1) Introduction of the definite article.

E steise wissemusîngin tawas, ênstan swintan noseilien, stan perôniskan, steise grijkan, steise tâwas, steise saûnas, steise swintan noseilîs, stêison grijkan. 
(2) Introduction of a possessive pronoun.

I nuson auschautins, II nouson anschautins, E nousons âuschautins.

I nuson auschantnikamans, II nouson anschautinekamans, E noûsons auschautenîkamans.

(3) Loss of inflection in the adjective.

I wismosingis thawas, II wyssen mukis thawas, E steise wissemusîngin tawas.

I swinte naseilis, II swyntas naseylis, E steise swintan noseilîs.

Lexical changes comprise the following:

I ny anterpinsquan menentwey, II nienbænden westwey, E ni enbândan westwei.

I pattiniskun, II salobisquan, E sallûban.

I falsch, II reddi, E redde.

I waitiatun, II waytiaton, E dâtwei.

I wismosing, II wissemokin, E wissemusîngin.

I patickots, II pagauts, E pogauts.

I preitickray, II preytickaray, E prei tickrômien.

I wismosingis, II wyssen mukis, E wissemusîngin.

I et werpsannan, II, E etwerpsennian.

I laeims, II ryeky, E rijks.

I perbandan, II perbandasnan, E perbandâsnan.

I menissnan, II menisnan, E pominisnan.

I att werpsannan, II etwerpsennian, E etwerpsennien.

I menisnan, II minisnan, E pominîsnan.

I conclude that the second catechism occupies an intermediate position between the first and the third. Since the three texts represent three distinct varieties of the Old Prussian language, they must be kept apart in an analysis of the material. The older catechisms reflect a linguistic system which is clearly more archaic than the language of the Enchiridion. They give us a glimpse of what Prussian must have looked like before it succumbed to the pervasive influence of German. The analysis offered here provides a temporal dimension which is crucial for a correct evaluation of the historical 
development.

\section{References}

Kortlandt, F. 1982. Innovations which betray archaisms, "Baltistica", 18/1, $4-9$.

Kortlandt, F. 1988. Van Wijk's Altpreussische Studien revisited, in Nicolaas van Wijk (1880-1941): A collection of essays on his life and work = Studies in Slavic and general linguistics, 12, Amsterdam: Rodopi, 237-243.

Kortlandt, F. 1998. The development of the Prussian language in the 16th century, in: Baltistik: Aufgaben und Methoden (ed. A. Bammesberger), Heidelberg: Carl Winter.

Van Wijk, N. 1918. Altpreussische Studien: Beiträge zur baltischen und zur vergleichenden indogermanischen Grammatik, Haag: Martinus Nijhoff.

\section{Senujų prūsų katekizmų kalba \\ Frederik Kortlandt (Leiden)}

Savo pranešime $1995 \mathrm{~m}$. Eichstätt'o konferencijoje autorius parodè, kad Enchiridiono kalba yra vèlyvesnis ankstesniuju katekizmu kalbu vystymosi rezultatas. Stipri vokiečiu kalbos sintaksès itaka yra logiškai laukiama neišvengiamoje kalbos mirties situacijoje. Idiosinkraziju nuoseklumas Will'o tekste aiškiai rodo jo neabejotiną prūsu kalbos mokejjimą. Siame straipsnyje autorius bando parodyti šitu atradimu pasekmes gramatinès sistemos analizei. Išreiškiama nuomonè, kad neteisingas istorinès raidos suvokimas iššaukia klaidingu prūsu kalbos priešistorijos hipoteziu gimimą. 